\title{
Molecular Dynamics Modeling of the Effect of Nanotwins on the Superelasticity of Single-Crystalline NiTi Alloys
}

\author{
Yang Guo, ${ }^{1}$ Xiangguo Zeng, ${ }^{1}$ Huayan Chen, ${ }^{1}$ Tixin Han, ${ }^{1}$ Heyi Tian, ${ }^{1}$ and Fang Wang ${ }^{2}$ \\ ${ }^{1}$ College of Architecture and Environment, Sichuan University, Chengdu 610065, China \\ ${ }^{2}$ Faculty of Materials and Energy, Southwest University, Chongqing 400715, China \\ Correspondence should be addressed to Xiangguo Zeng; xiangguozeng@scu.edu.cn and Fang Wang; wangfang_cq1978@163.com
}

Received 19 May 2017; Accepted 18 July 2017; Published 17 September 2017

Academic Editor: Jörg M. K. Wiezorek

Copyright (c) 2017 Yang Guo et al. This is an open access article distributed under the Creative Commons Attribution License, which permits unrestricted use, distribution, and reproduction in any medium, provided the original work is properly cited.

\begin{abstract}
The objective of this work is to simulate the superelasticity and shape-memory effect in a single-crystalline nickel-titanium (NiTi) alloy through a molecular dynamics (MD) study. Cooling and heating processes for this material are reproduced to investigate the temperature-induced phase transformation in its microstructure. It is found that the martensitic transformation and its reverse process occur accompanied by an abrupt volume change, and the transformed variants lead to the appearance of the (001) type compound twin. In addition, the transform temperatures for martensite start $\left(M_{s}\right)$ and austenite finish $\left(A_{f}\right)$ are determined, respectively. The results indicate that when the temperature is beyond $A_{f}$ during the compressive loadingunloading, the superelastic behavior becomes pronounced, which is attributed to the role of nanotwins on the transformation from the austenitic phase (B2) to martensitic phase $\left({\mathrm{B} 19^{\prime}}^{\prime}\right)$. Compared to existing experimental data, a reasonable agreement is achieved through the modeling results, highlighting the importance of the compound twins for dominating the superelasticity of nanostructured NiTi alloys.
\end{abstract}

\section{Introduction}

Shape-memory alloys (SMAs) have important applications involving miniactuators, microelectromechanical systems, robotics, biomedicine, and even smart clothing [1] because of their ability of recovering their original shape under heating conditions and sustaining large elastic strains. Such two behaviors are recognized as the shape-memory effect (SME) and the superelasticity (SE), respectively. In recent years, these alloy materials are widely accepted and used as a functional material in the field of the automotive and aerospace industries. Among the many different types of SMAs, a nickel-titanium (NiTi) alloy has gained widespread attention owing to its excellent inherent advantages, such as mechanical properties, corrosion resistance, biocompatibility, and phase transformation close to room temperature. There is an evidence that demonstrates that the SME and SE in the NiTi alloy are caused by the martensitic transition (MT) and its reversible one (i.e., austenitic transition (AT)) between cubic B2 (austenite) and monoclinic B19' (martensite), respectively [2], which are induced by environmental temperature and applied stress. To meet the specific requirements of safe design, whether and how such behaviors occur in very small length scale system are of growing interest.

In the last decade, much effort had been focused on investigating the SME and SE in the NiTi alloy at the nanoscale [2]. Based on experimental observations, it was shown that a stress-induced reversible transformation between the two ordered phases resulted in the strain recovery under isothermal conditions [3]. A constitutive law containing the superelastic effect subjected to multiaxial loading was obtained through direct mechanical experiments [4]. In situ X-ray diffraction was employed to record the atomic configuration of phase transformations during tensile loading-unloading [5]. With regard to the temperature-induced phase transformation, the grain size dependence of phase transition temperature was observed through the transmission electron microscopy (TEM) [6]. Besides, it is justified that atomistic simulations can be regarded as a tool for investigating the MT, SME, and SE by providing more structural details and 
mechanistic insights at the atomic scale. For example, MD simulations exhibit a possibility of describing the phase structures derived from temperature- and stress-induced phase transformation $[7,8]$. In the MD method, several characteristics of phase transformation in the NiTi alloy had been revealed through a semiempirical pair potential [9]. Liang and Zhou [10] conducted an atomistic simulation and successfully achieved the SME in single-crystalline fcc $\mathrm{Cu}$ nanowires. It was found that when a thermomechanical load was applied in a martensitic NiAl system, the total recovery of deformation could be obtained by MD under the condition of periodic boundaries [11]. Considering the complexity of microstructure of martensite, the semiempirical potentialbased atomistic simulation approach is expected to play an important role in bridging a link between experiments and numerical models for understanding the transformation mechanism in SMAs [12-14].

It is well known that the evolution of twinning behavior is of great importance for the martensitic phase transformation in SMAs [3]. Twin martensites are conventionally classified into three categories: type I (i.e., the twin plane is a rational crystal plane), type II (i.e., the twin shear is a rational crystallographic direction), and compound twin (both the twin plane and twin shear are rational), the detailed definitions of which are described in the review [15]. Krishnan and Singh found a new type of $\mathrm{B} 19^{\prime}$ martensite in the thermally cycled $\mathrm{NiTi}$ alloy, and the (001) compound twin was shaped [16]. Also, the occurrence and disappearance of microtwins had been confirmed by in situ TEM during mechanical cycles [17]. Some reports claimed that the stress-induced martensite transformation would occur accompanied with deformation twinning when the alloy was subjected to plastic deformation in the austenite state [18]. Zhong et al. performed an in-depth study on the structure and geometrical limit of nanoscale twins in NiTi by combining the crystallographic theory with atomistic simulations [19]. However, the role of atomic interactions involved during structural transformations, such as twinning and size effect as well as the temperature-dependent behavior, is not yet well understood. Moreover, a physical mechanism is still required to explain the characteristics of both temperature- and stress-induced phase transformations.

The numerical simulation performed in the present work differs from those of other studies. The motivation behind previous investigations has been to study phase transformations in a single-crystalline NiTi alloy under uniaxial compression loading. It should be emphasized that our investigation is primarily focused on conducting numerical analysis for interatomic motions by using MD simulations coupled with an effective potential [21]. Considering that the twinning structure always appears inside the gain, an initial model is prepared as a single crystal NiTi with the B2 structure. Compared with available experimental information, the role of nanotwins in the atomistic behavior is further examined as well. This study is expected to provide a more comprehensive understanding of the temperature- and stress-induced phase transformation mechanisms for this kind of material from the perspective of atomic scale.

\section{Materials and Methods}

2.1. 2NN MEAM Potential. It is widely accepted that the embedded-atom method (EAM) can be employed to characterize the interatomic potential [27]. Besides, a modified embedded-atom method (MEAM) combined with an angular dependent term has been proposed by Baskes [22]. Comparatively speaking, MEAM potentials are well suited for simulations of multicomponent systems composed of elements with different ground states, because they allow for characterizing a wide range of phases ( $f c c, b c c, h c p$, diamondstructured, and even gases) using a common mathematical function [28]. However, Ko et al. [21] strongly recommended the use of second nearest-neighbor modified embeddedatom method (2NN MEAM) when choosing an interatomic potential in the NiTi binary alloy. Furthermore, a comparison of the predicted results with the experimental ones [3] indicated that such a model could be available for representing the characteristics of the low temperature martensitic structure $\left(\mathrm{B} 19^{\prime}\right)$.

The total potential energy of the 2 NN MEAM is expressed as follows:

$$
E=\sum_{i}\left[F_{i}\left(\bar{\rho}_{i}\right)+\frac{1}{2} \sum_{j \neq i t} s_{i j} \phi_{i j}\left(R_{i j}\right)\right],
$$

where $F_{i}$ is the embedding energy, which is a function of background electron density $\bar{\rho}_{i} . S_{i j}$ is the screening function and $\phi_{i j}\left(R_{i j}\right)$ is the pair interaction between atoms $i$ and $j$ separated by a distance $R_{i j}$. In fact, what the nicest feature the improved MEAM has is that it accounts for the second nearest-neighbor interactions when calculating the embedding energy, while the original MEAM merely considers the first nearest-neighbor interactions through a strong manybody screening function. A detailed formulation of the $2 \mathrm{NN}$ MEAM formalism was provided in literature $[29,30]$.

Figure 1 shows the calculated cohesive energy $E_{c}$ (eV/atom) and equilibrium lattice constants $a_{0}(\AA)$ of the B2 structure under the framework of $2 \mathrm{NN}$ MEAM potential.

When the lattice constant varies from 2.5 to $4 \AA$, the atom energy for each instantaneous equilibrium state is calculated, and then the cohesive energy is achieved by minimizing all the atom energies. In this work, the computed energy is $5.05 \mathrm{eV}$ corresponding to an equilibrium lattice constant of $a_{0}=2.999 \AA$, and these values conform to other potentials [20]. Some other calculated physical properties of equiatomic NiTi compounds are presented in Table 1. Compared with experimental data and other potentials, it is demonstrated that the parameters obtained from such a potential could be used in our subsequent simulations for investigating the phase transformations induced by temperature and stress.

2.2. Physical Structures. For the equiatomic NiTi alloy, the geometric structure of austenitic phase is the cubic B2 (with space group of $P m \overline{3} m$ ) structure, while the $\mathrm{B} 19^{\prime}$ structure (with space group of $P 2_{1} / \mathrm{m}$ ) is remarkably characterized by a monoclinic angle $\left(\beta=96.8^{\circ}\right)$ and shuffling of $\mathrm{Ni}$ and $\mathrm{Ti}$ atoms on the $(110)_{\mathrm{B} 2}$ plane [3]. Figure 2 shows the lattice structures for $\mathrm{B} 2$ and $\mathrm{B}^{\prime} 9^{\prime}$, respectively. 
TABLE 1: Comparison of calculated and experimental physical constants for the B2 phase.

\begin{tabular}{|c|c|c|c|c|c|}
\hline Proper & & 2NN MEAM potential & Experiments & Literature potential [22] & DFT simulations \\
\hline \multirow{4}{*}{ Elastic constants $(\mathrm{GPa})$} & C11 & 143 & $162[23]$ & 206 & $138[24]$ \\
\hline & $\mathrm{C} 12$ & 128 & $129[23]$ & 136 & $169[24]$ \\
\hline & $\mathrm{C} 44$ & 80 & $35[23]$ & 47 & $40[24]$ \\
\hline & Bulk modulus, B & 133 & $140[23]$ & 159 & $159[24]$ \\
\hline \multicolumn{2}{|c|}{ Cohesive energy $(\mathrm{eV})$} & -5.05 & $-4.95[25]$ & -5.022 & $-4.99[26]$ \\
\hline \multicolumn{2}{|c|}{ Lattice constant $(\AA)$} & 2.999 & $3.01[25]$ & 3.008 & $2.96[26]$ \\
\hline
\end{tabular}

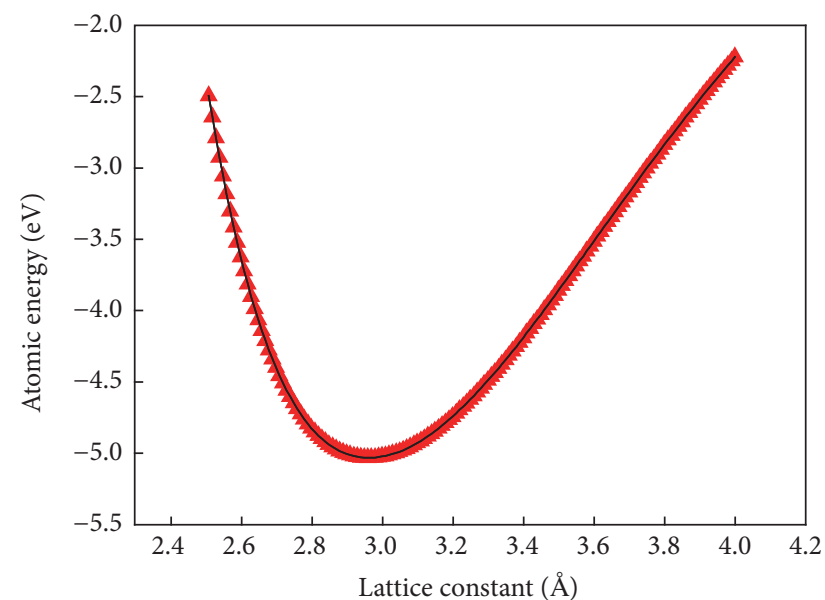

Figure 1: Atomic energy $E_{c}(\mathrm{eV})$ versus unit cell lattice constant $a_{0}$ $(\AA)$.
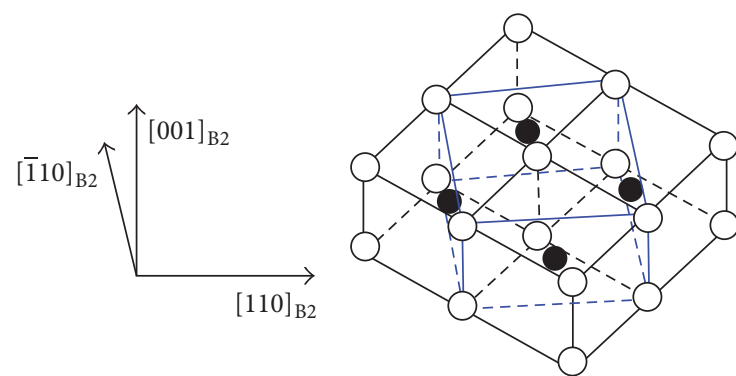

B2
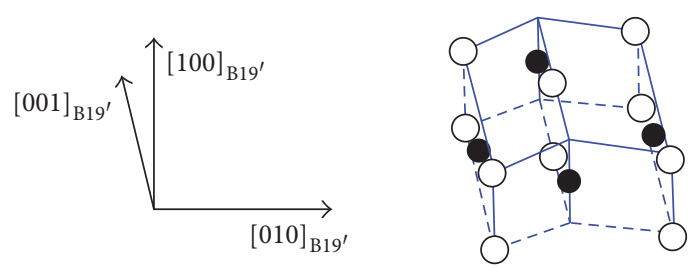

$\mathrm{B} 19^{\prime}$

$\bigcirc \mathrm{Ti}$

FIGURE 2: Schematic of the structure of $\mathrm{B} 2$ and $\mathrm{B} 19^{\prime}$.

2.3. MD Simulations. In this study, the open source software LAMMPS (Large scale Atomic/Molecular Massively Parallel Simulator) [25] developed at Sandia National Laboratories, USA (http://lammps.sandia.gov/), is used to conduct MD simulations. The atomic configuration viewer Ovito [24] (http://ovito.org/) is utilized to analyze microstructural evolutions during phase transformations, and local atomic arrangements are visualized by the common neighbor analysis (CNA) [7].

Firstly, the single crystal of B2 type unit cells (with $a_{0}=$ $2.999 \AA$ ) was produced by the atomic positions of the basis

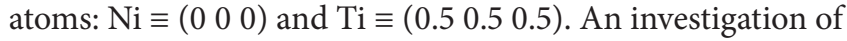
the temperature-induced phase transition was performed to study the martensitic transformation mechanism. The initial model was set as the B2 structure, with the length of 20 cells, width of 20 cells, and thickness 5 cells. The total atoms were 4000. Several corresponding MD simulations were employed starting with supercells ranging in sizes from 4000 to 16000 atoms. The single crystal supercell of B2 structure was firstly equilibrated at $550 \mathrm{~K}$, using an isobaric-isothermal ensemble (NPT) at zero pressure. All the simulations were carried out in the temperature range from 550 to $50 \mathrm{~K}$ with a cooling rate of $1 \mathrm{~K} / \mathrm{ps}$ and heating from 50 to $550 \mathrm{~K}$ with the same rate. Periodic boundary conditions were applied along all three dimensions for the purpose of computational efficiency [26]. Cell dimensions, cell angles, and individual atomic positions were allowed to relax. Then, the changes in atomic volumes were carefully recorded to observe the occurrence of phase transitions.

For the purpose of simplicity only, a pillar was prepared as a single crystal consisting of the B2 structure with dimensions of $9.0 \times 8.5 \times 2.1 \mathrm{~nm}$ and 12000 atoms when simulating the stress-induced phase transition. The sides of the pillar were $\{110\}$-type surfaces, and the longitudinal direction was aligned to the [001] direction in the structure. After these steps, the transition temperatures under a zero-stress state were obtained, and then $M_{s}$ and $A_{f}$ were calculated to be 240 and $440 \mathrm{~K}$, respectively. After that, the pillar was equilibrated to $450 \mathrm{~K}$. It was emphasized that this temperature was slightly higher than $A_{f}(440 \mathrm{~K})$, which could be responsible for maintaining the B2 phase under a zero-stress state. Finally, a compressive loading was applied by adjusting the stress along the longitudinal direction of the samples. Under the temperature of $450 \mathrm{~K}$, the compressive stresses increases from 0 to $2.1 \mathrm{GPa}$ and completely decreases. A constant loading rate of $\pm 3.5 \mathrm{MPa} / \mathrm{ps}$ was maintained. $\mathrm{MD}$ simulations were performed using a time step of $2 \mathrm{fs}$.

\section{Results and Discussion}

3.1. Temperature-Induced Phase Transition. Figure 3 shows the temperature dependence of atomic volumes for a cell combined with 4000 atoms. It is evident that the obtained 


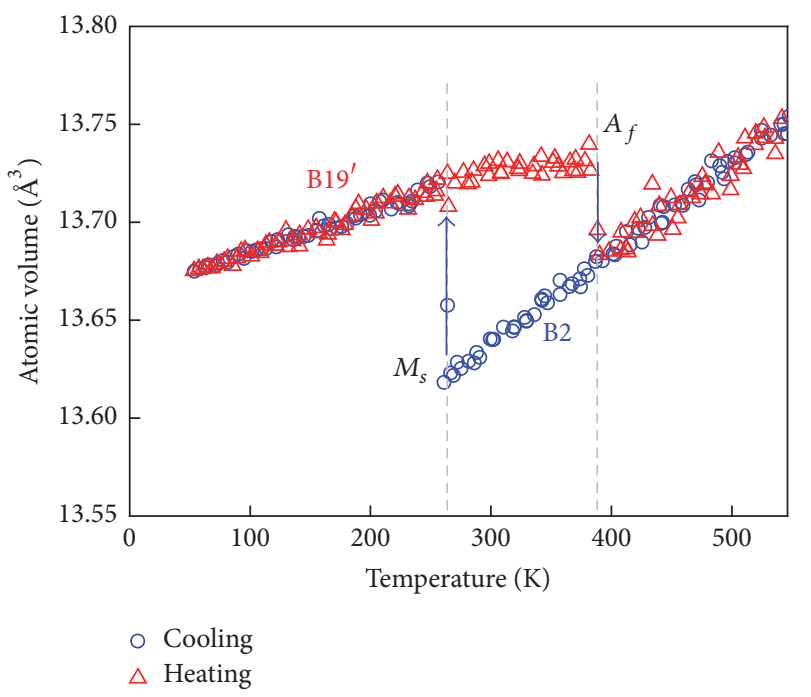

FIGURE 3: The atomic volume dependence on temperature during cooling (blue circle) and heating (red triangle) process for NiTi alloy with 4000 atoms. The discrete jumps indicated by the arrows represent the occurrence of phase transitions.

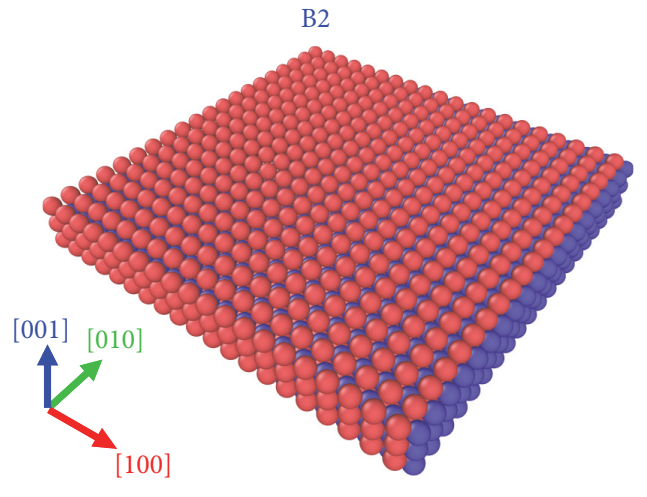

(a)

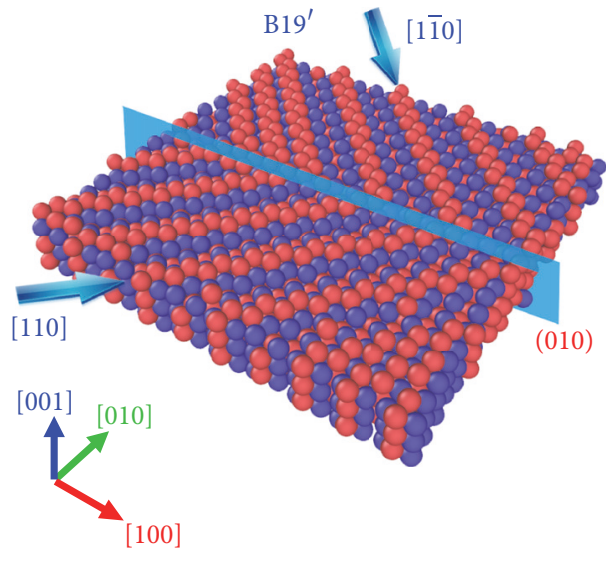

(b)

Figure 4: Atomic structures of (a) the initial B2 phase and (b) the transformed B19' phase including (001) compound twin boundaries. Ni and $\mathrm{Ti}$ atoms are represented by blue and red, respectively. Note that the twinning direction and twinning plane (010) are showed by the coordinate of B2 phase.

volumes for the austenitic and martensitic structures at room temperature $(\sim 300 \mathrm{~K})$ are $13.64 \AA^{3}$ and $13.73 \AA^{3}$, respectively, which are in good accordance with those of experiment values (13.70 $\AA^{3}$ and $13.80 \AA^{3}$ ) [31]. From this figure, it is distinctly demonstrated that the martensite start temperature $\left(M_{s}\right)$ is $270 \mathrm{~K}$ from the discrete positive change, which yield a closer fit to the experimental data when performing a comparison with the previous study [20]. Besides, the discrete decrease during the reheating process marks that the martensite phase transforms back into the austenite at the temperature of $390 \mathrm{~K}$. It is obvious that both martensitic and reverse transformation (i.e., austenite) occur in the process of cooling and heating. The physical reason hiding behind such phenomena is believed to be due to the difference of atomic volume between B2 and B19'.

Figure 4 depicts atomic configurations of a simulated supercell before and after a martensitic transition. As shown in Figure 4(b), a twinned structure with finely dispersed compound twin boundaries (001) is observed in the transformed martensite. In previous studies, it was found that such a behavior does exist in the nanocrystalline structure [32].

Figure 5(a) shows the transition temperatures calculated using the 16000 -atom supercell. From the results, $M_{s}$ is about $255 \mathrm{~K}$, which is lower than the one $(270 \mathrm{~K})$ of the 4000 atom supercell. This could be attributed to the fact that the undercooling shows an increasing trend with enlarging 


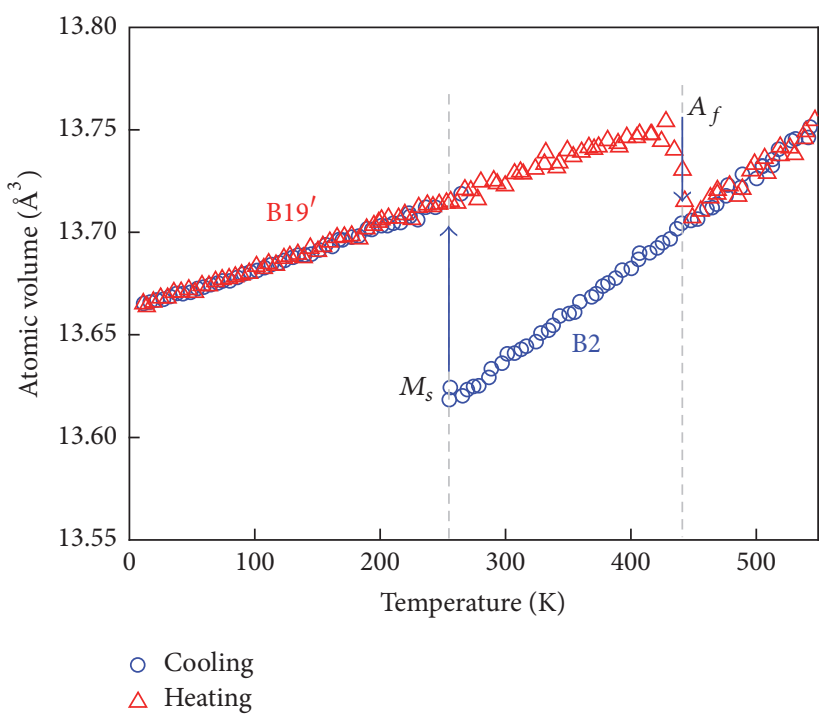

(a)

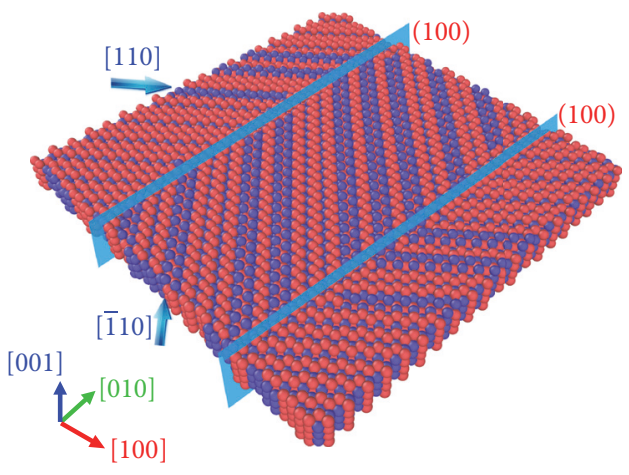

(b)

FIGURE 5: (a) The atomic volume dependence of temperature during cooling and heating and (b) the twinned martensite B19' phase with 16000 atoms.

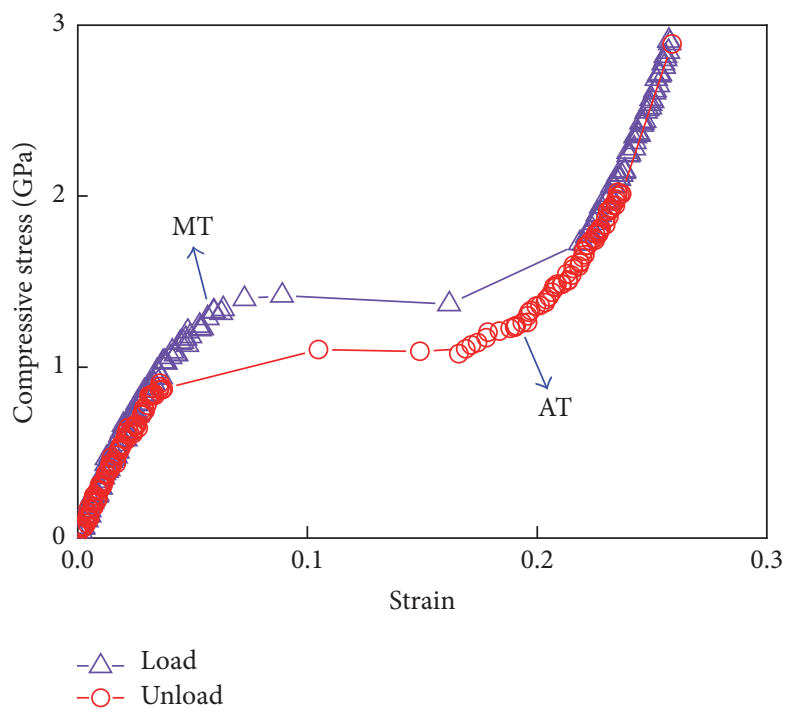

FIGURE 6: Simulated stress-strain response of a single crystal NiTi at $450 \mathrm{~K}$. Note that the positive values mean the compressive strain values.

the supercell sizes. Compared with the 4000-atom supercell $(390 \mathrm{~K}), A_{f}$ is found to be a higher value of $445 \mathrm{~K}$ of 16000 atoms. Furthermore, the larger supercell exhibits a considerably wider thermal hysteresis, which is on account of the increasing of the number of atoms in twin zones. Figure 5(b) shows that more compound twins emerge in the 16000-atom supercell, accompanied by a twinning direction of [010]. These results indicate that the twin direction is not fixed but could vary with cell sizes. Additionally, it is evident that the average width of twin is in nanometer magnitude, which agrees well with experimental data [32]. However, it must be pointed out that the size effect on the transformation temperatures and the nanotwin formations disappears when the specimen is sufficiently large [21].

\subsection{Stress-Induced Phase Transition}

3.2.1. Stress-Strain Behavior. Figure 6 illustrates a resultant stress-strain response of the pillar subjected to longitudinal compressive deformations at $450 \mathrm{~K}$. In this figure, the phase transition onset in loading/unloading is represented by an arrow. It is clear that the stress shows an increasing tendency with the applied strain at the beginning of loading, which is known as the elastic stage of martensitic structure. Due to the 

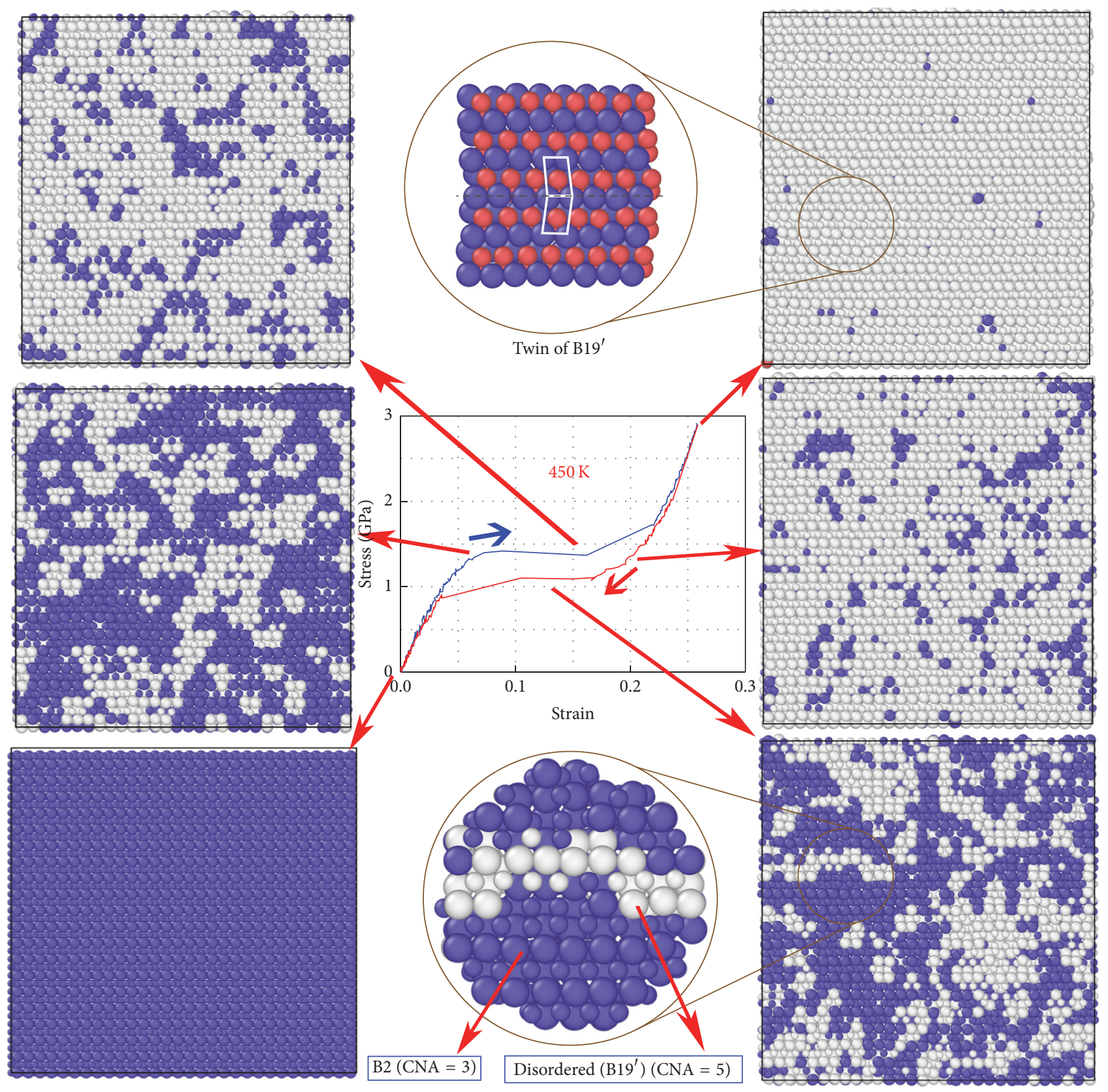

FIGURE 7: Snapshots of the nano-NiTi during the compressive loading and the unloading process. The color of the atoms is scaled according to the common neighbor analysis (CNA) pattern [20]. In each snapshot, blue atoms correspond to the B2 structure and gray atoms to the $\mathrm{B} 19^{\prime}$ structure. A few of the outmost surface layers (thickness of $0.5 \mathrm{~nm}$ ) are not visualized for clarity.

occurrence of phase transformation from B2 to $\mathrm{B} 19^{\prime}$, a plateau appears in the stress-strain curve. The phase transformation occurred at the stress level of $1339 \mathrm{MPa}$, which was in agreement with the experimental data $(1 \mathrm{GPa})$ achieved from in situ TEM tests [33]. As the applied load increases, another elastic stage (austenite) emerges with the end of martensitic transformation. During the unloading process, B19' will directly back into B2. From the figure, it is shown that the full recovery of the initial B2 structure arises after a load cycle, which exhibits the superelastic behavior. In other words, the $\mathrm{NiTi}$ alloy allows for sustaining large elastic strains.
3.2.2. Reversible Phase Transformation. Figure 7 shows the atomistic snapshots of the NiTi single crystal during various deformation stages at $450 \mathrm{~K}$. For the purpose of hiding the periodic atoms on the boundaries, a few of the outmost surface layers are not visualized. As expected, only the elastic compression deformation occurs at the initial elastic strain (from 0 to about 5\%). In other words, the crystal structure remains unchanged, which is similar to the initial structure (with B2 unit cells). When the stress level reaches $1339 \mathrm{MPa}$, it seems that the atoms take a considerable rearrangement. Some austenitic lattices (with B2 unit cells) gradually 


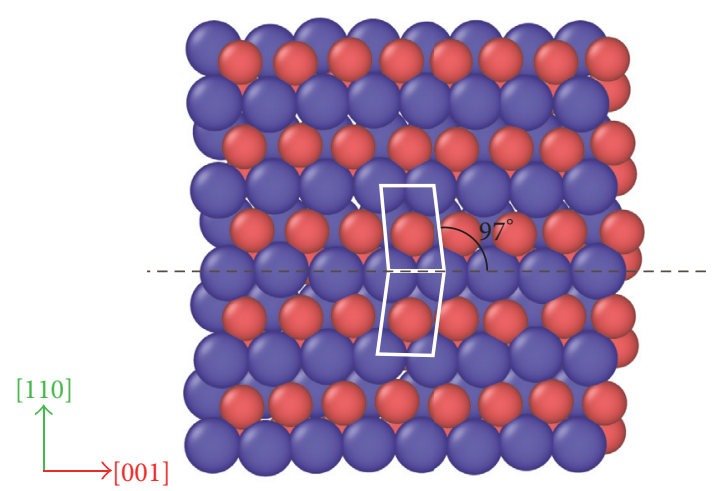

Figure 8: Atomic structure of (110) compound twins, and the white parallelogram indicates a monoclinic unit cell with the monoclinic angle $\beta \approx 97^{\circ}$. The mirror twin plane (dashed line) is located on the pure Ni layer. A few of the outmost surface layers (thickness of $0.5 \mathrm{~nm}$ ) are not visualized for clarity. $\mathrm{Ni}$ and $\mathrm{Ti}$ atoms are represented by blue and red, respectively.

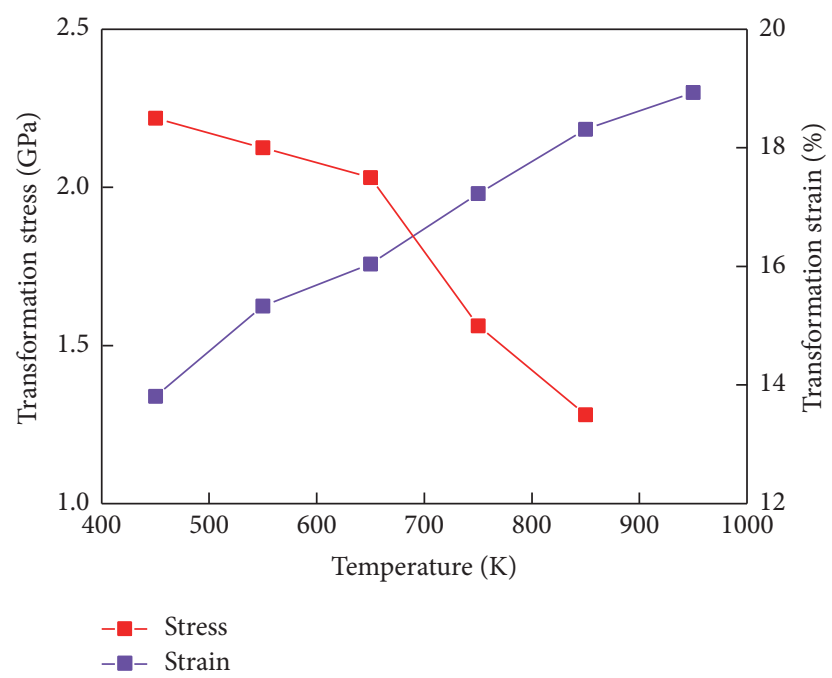

FIGURE 9: Calculated values of transformation stress and strain for the NiTi austenite compressed in $\langle 001\rangle_{\mathrm{B} 2}$ direction when the temperature ranges from 450 to $950 \mathrm{~K}$.

transform into the monoclinic martensitic lattices. With continuing compression, the volume fraction of $\mathrm{B} 19^{\prime}$ increases. The martensitic transformation is verified by a careful inspection for the lattice structure within the phase transformation regions, which is illustrated in Figure 8. In examining this figure, the monoclinic unit cell structure with monoclinic angle $\beta=97^{\circ}$ is in stark contrast with the $\mathrm{B} 2$ lattice. Moreover, the newly transformed ${\mathrm{B} 19^{\prime}}^{\prime}$ variants along different directions create multiple mirrored strips, that is, the twinned structures. This phenomenon is the direct evidence of the (001) type compound twins of $\mathrm{B}^{\prime} 9^{\prime}$, consistent with the behavior observed by TEM [16].

The internal twinning behavior continues until the crystal fully converts into the martensitic phase (with internally twinned ${\mathrm{B} 19^{\prime}}^{\prime}$ lattice), which occurs at about 20\% applied strain. Afterwards, the continuation of applied loading leads to an elastic strain of the twinned martensite, corresponding to the rising portion of stress-strain curve (from 20 to $26 \%$ ). The final crystal structure (at about 26\% applied strain) is characterized by multiple internal twins, seen in Figure 7.

During the unloading, the deformation in the crystal embedded with twinned martensite fully recovers when the total strain is $26 \%$, which may be expected as a result of a combination of elastic relaxation and a reverse phase transformation (from B19' to B2). In Figure 7, the atomistic snapshots indicate a gradual reduction in volume fractions of the twinned $\mathrm{B} 19^{\prime}$, and $\mathrm{B} 19^{\prime}$ diminishes at the lower stress plateau. It is suggested that the internally twinned martensitic structure remains stable only when the stress level is high. However, the removal of applied stress serves to cause the phase transformation from $\mathrm{B} 19^{\prime}$ to $\mathrm{B} 2$.

\subsubsection{Dependency of Transformation Stress on Temperature.} The relationship between the environmental temperature and transformation stress/strain under stress-controlled compressive loading is provided in Figure 9, which shows that, based on $0.2 \%$ offset method, the transformation stress increases from 1339 to $2300 \mathrm{MPa}$ as the temperature increases from 450 to $950 \mathrm{~K}$, while the corresponding strain decreases from 18.5 to $13.6 \%$ for the same temperature magnitude. These observations from the simulations are in general agreement with experimental findings [3]. The temperature effect could be rationalized on the basis of the thermodynamics of transforming alloys [12]. The twinned monoclinic B19' structure that constitutes the martensitic phase becomes unstable at an elevated temperature. In order to complete the phase transformation from $\mathrm{B} 2$ to $\mathrm{B} 19^{\prime}$, an additional driving force is needed to be applied. As a result, the transformation stress level continues to increase with increasing temperature, which is absolutely different from any conventional alloy that would undergo a decreasing flow stress. When the temperature is sufficiently high (above $950 \mathrm{~K}$ ), any transformation strain no longer appears in the parent B2 behaviors, demonstrating the temperature-dependent plastic deformation of austenitic phase.

\section{Conclusions}

Compared with previous studies, a deeper understanding of compression in the NiTi alloy is gained by crystallographic calculations on the formation of nanotwinned martensitic structures in a single-crystalline NiTi alloy in the present work. The 2 NN MEAM potential is testified by a comparison of the calculated properties and experimental results, such as elastic constant, melting temperature, cohesive energy, and equilibrium lattice constant. Molecular dynamics method is employed to investigate the stress-induced and temperatureinduced martensitic phase transformation in NiTi at the nanometer scale. The simulations indicate that the twinning structure has a crucial impact on the phase transformation temperature. The martensite start temperature $\left(M_{s}\right)$ and austenite finish temperature $\left(A_{f}\right)$ of the NiTi alloy are obtained and found to provide a reasonable agreement with experimental data. In addition, the superelastic behavior is reproduced through numerical modeling. From the results of 
the stress-induced phase transition under the $\langle 100\rangle_{\mathrm{B} 2}$ compressive loading, it could be concluded that a full recovery of the shape occurs after a loading-unloading cycle. Also, the results demonstrate that the stress-induced phase transformation (from austenite to martensite) does exist in single crystal NiTi alloy, the size of which is below $100 \mathrm{~nm}$. This phenomenon was previously observed by in situ TEM nanocompressive tests. The atomic screenshots of the compression specimen indicate that there are multiple $\mathrm{B}^{\prime} 9^{\prime}$ variants of small misorientations with each other and they form the nanotwins. Importantly, the relationship between the environmental temperature and transformation behavior is established through numerical simulations. However, a more precise calibration of the model, capable of accounting for a polycrystalline NiTi alloy and its cyclic behavior, would have to be considered in further study.

\section{Conflicts of Interest}

The authors declare that there are no conflicts of interest regarding the publication of this paper.

\section{Acknowledgments}

The authors gratefully acknowledge the financial support of NSAF under Grant no. U1430119. The authors would also like to express their gratitude to Professor Jun Chen, Institute of Applied Physics and Computational Mathematics, Beijing, China, for helpful discussions.

\section{References}

[1] J. M. Jani, M. Leary, A. Subic, and M. A. Gibson, "A review of shape memory alloy research, applications and opportunities," Materials \& Design, vol. 56, pp. 1078-1113, 2014.

[2] T. Waitz, K. Tsuchiya, T. Antretter, and F. D. Fischer, "Phase transformations of Nanocrystalline martensitic materials," MRS Bulletin, vol. 34, no. 11, pp. 814-821, 2009.

[3] K. Otsuka and X. Ren, "Physical metallurgy of Ti-Ni-based shape memory alloys," Progress in Materials Science, vol. 50, no. 5, pp. 511-678, 2005.

[4] J. M. McNaney, V. Imbeni, Y. Jung, P. Papadopoulos, and R. O. Ritchie, "An experimental study of the superelastic effect in a shape-memory Nitinol alloy under biaxial loading," Mechanics of Materials, vol. 35, no. 10, pp. 969-986, 2003.

[5] A. Ahadi and Q. Sun, "Stress-induced nanoscale phase transition in superelastic NiTi by in situ X-ray diffraction," Acta Materialia, vol. 90, pp. 272-281, 2015.

[6] T. Waitz, T. Antretter, F. D. Fischer, and H. P. Karnthaler, "Size effects on martensitic phase transformations in nanocrystalline NiTi shape memory alloys," Materials Science and Technology, vol. 24, no. 8, pp. 934-940, 2008.

[7] D. Mutter and P. Nielaba, "Simulation of the thermally induced austenitic phase transition in NiTi nanoparticles: simulation of phase transitions in NiTi nanoparticles," The European Physical Journal B, vol. 84, no. 1, pp. 109-113, 2011.

[8] R. Mirzaeifar, K. Gall, T. Zhu, A. Yavari, and R. Desroches, "Structural transformations in NiTi shape memory alloy nanowires," Journal of Applied Physics, vol. 115, no. 19, Article ID 194307, 2014.
[9] J. Deng, X. Ding, T. Lookman et al., "Microscopic mechanism of martensitic stabilization in shape-memory alloys: Atomic-level processes," Physical Review B - Condensed Matter and Materials Physics, vol. 81, no. 22, Article ID 220101, 2010.

[10] W. Liang and M. Zhou, "Atomistic simulations reveal shape memory of fcc metal nanowires," Physical Review B - Condensed Matter and Materials Physics, vol. 73, no. 11, Article ID 115409, 2006.

[11] Z. L. Xie and Y. Liu, "HRTEM study of (011) type II twin in NiTi shape memory alloy," Philosophical Magazine, vol. 84, no. 32, pp. 3497-3507, 2004.

[12] J. G. Boyd and D. C. Lagoudas, "A thermodynamical constitutive model for shape memory materials. Part I. The monolithic shape memory alloy," International Journal of Plasticity, vol. 12, no. 6, pp. 805-842, 1996.

[13] A. Sengupta and P. Papadopoulos, "Constitutive modeling and finite element approximation of B2-R-B19' phase transformations," in in Nitinol polycrystals, vol. 198, pp. 3214-3227, Computers Methods in Applied Mechanics Engineering, 2009.

[14] C. H. Lei, L. J. Li, Y. C. Shu, and J. Y. Li, "Austenite-martensite interface in shape memory alloys," Applied Physics Letters, vol. 96, no. 14, Article ID 141910, 2010.

[15] J. W. Christian and S. Mahajan, "Deformation twinning," Progress in Materials Science, vol. 39, no. 1-2, pp. 1-157, 1995.

[16] M. Krishnan and J. B. Singh, "A novel B19' martensite in nickel titanium shape memory alloys," Acta Materialia, vol. 48, no. 6, pp. 1325-1344, 2000.

[17] L. C. Zhao, Y. F. Zheng, and W. Cai, "Study of deformation micromechanism in cold-deformed TiNi based alloys," Intermetallics, vol. 13, no. 3-4, pp. 281-288, 2005.

[18] T. Ezaz, J. Wang, H. Sehitoglu, and H. J. Maier, "Plastic deformation of NiTi shape memory alloys," Acta Materialia, vol. 61, no. 1, pp. 67-78, 2013.

[19] Y. Zhong, K. Gall, and T. Zhu, "Atomistic study of nanotwins in NiTi shape memory alloys," Journal of Applied Physics, vol. 110, no. 3, Article ID 033532, 2011.

[20] G. Ren and H. Sehitoglu, "Interatomic potential for the NiTi alloy and its application," Computational Materials Science, vol. 123, pp. 19-25, 2016.

[21] W.-S. Ko, B. Grabowski, and J. Neugebauer, "Development and application of a Ni-Ti interatomic potential with high predictive accuracy of the martensitic phase transition," Physical Review B - Condensed Matter and Materials Physics, vol. 92, no. 13, Article ID 134107, 2015.

[22] M. I. Baskes, "Modified embedded-atom potentials for cubic materials and impurities," Physical Review B, vol. 46, no. 5, pp. 2727-2742, 1992.

[23] A. C. Stott, P. B. Abel, C. Dellacorte, S. V. Pepper, and D. A. Dixon, "Computational studies of the NiTi alloy system: bulk, supercell, and surface calculations," MRS Proceedings, vol. 1295, pp. 15-20, 2011.

[24] A. Stukowski, "Visualization and analysis of atomistic simulation data with OVITO-the Open Visualization Tool," Modelling \& Simulation in Materials Science \& Engineering, vol. 18, Article ID 015012, 2009.

[25] S. Plimpton, "Fast parallel algorithms for short-range molecular dynamics," Journal of Computational Physics, vol. 117, no. 1, pp. 1-19, 1995.

[26] W.-S. Ko, S. B. Maisel, B. Grabowski, J. B. Jeon, and J. Neugebauer, "Atomic scale processes of phase transformations in nanocrystalline NiTi shape-memory alloys," Acta Materialia, vol. 123, pp. 90-101, 2017. 
[27] M. S. Daw and M. I. Baskes, "Embedded-atom method: derivation and application to impurities, surfaces, and other defects in metals," Physical Review B, vol. 29, no. 12, pp. 6443-6453, 1984.

[28] B.-J. Lee and M. I. Baskes, "Second nearest-neighbor modified embedded-atom-method potential," Physical Review B - Condensed Matter and Materials Physics, vol. 62, no. 13, pp. 85648567, 2000.

[29] B. J. Lee, M. I. Baskes, H. Kim, and Y. K. Cho, "Second nearestneighbor modified embedded atom method potentials for bcc transition metals," Physical Review B, vol. 64, Article ID 184102, 2001.

[30] B. J. Lee, W. S. Ko, H. K. Kim, and E. H. Kim, “The modified embedded-atom method interatomic potentials and recent progress in atomistic simulations," Calphad-computer Coupling of Phase Diagrams \& Thermochemistry, vol. 34, pp. 510-522, 2010.

[31] S. D. Prokoshkin, A. V. Korotitskiy, V. Brailovski, S. Turenne, I. Y. Khmelevskaya, and I. B. Trubitsyna, "On the lattice parameters of phases in binary Ti-Ni shape memory alloys," Acta Materialia, vol. 52, no. 15, pp. 4479-4492, 2004.

[32] T. Waitz, "The self-accommodated morphology of martensite in nanocrystalline NiTi shape memory alloys," Acta Materialia, vol. 53, no. 8, pp. 2273-2283, 2005.

[33] J. Ye, R. K. Mishra, A. R. Pelton, and A. M. Minor, "Direct observation of the NiTi martensitic phase transformation in nanoscale volumes," Acta Materialia, vol. 58, no. 2, pp. 490-498, 2010. 

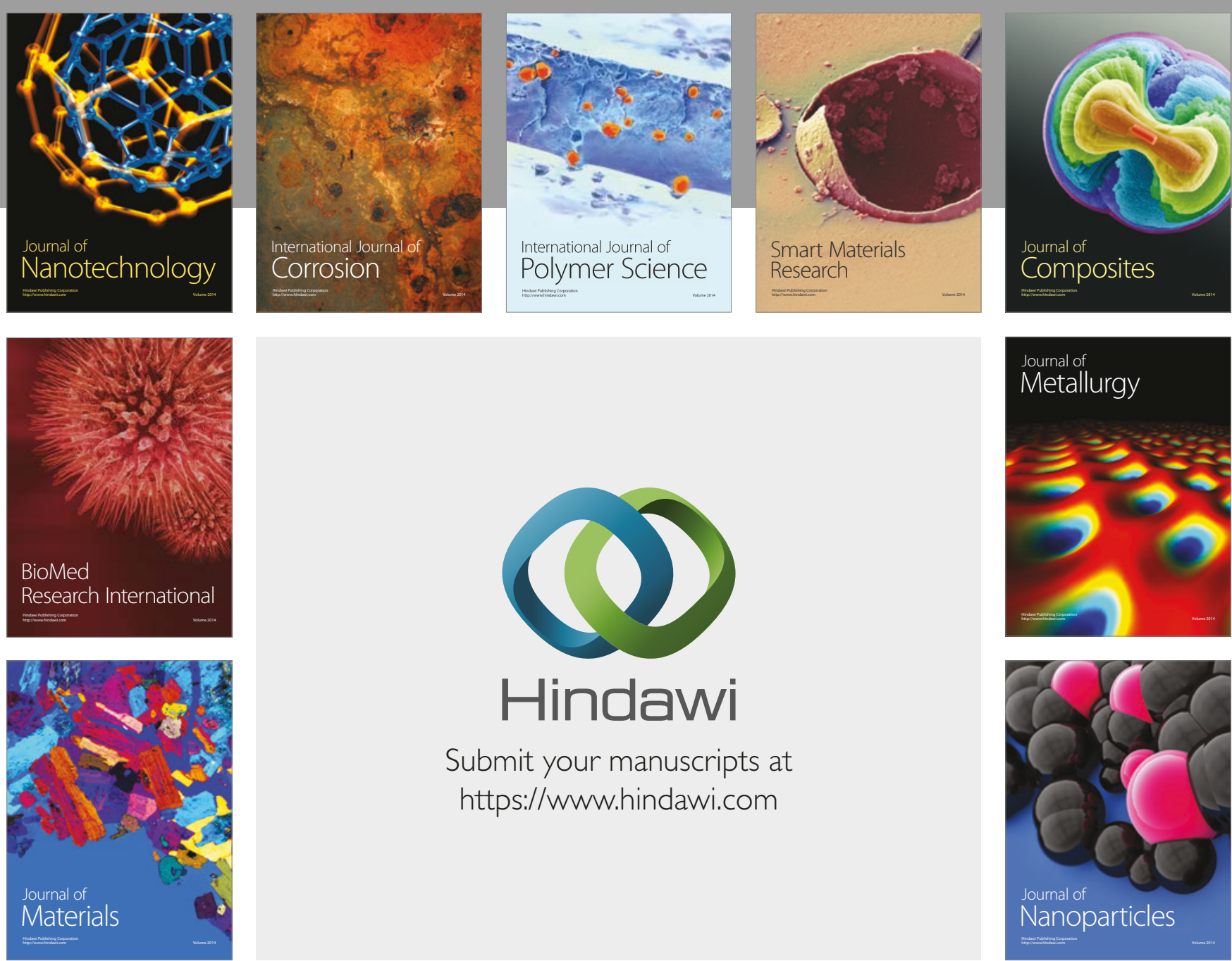

\section{Hindawi}

Submit your manuscripts at

https://www.hindawi.com
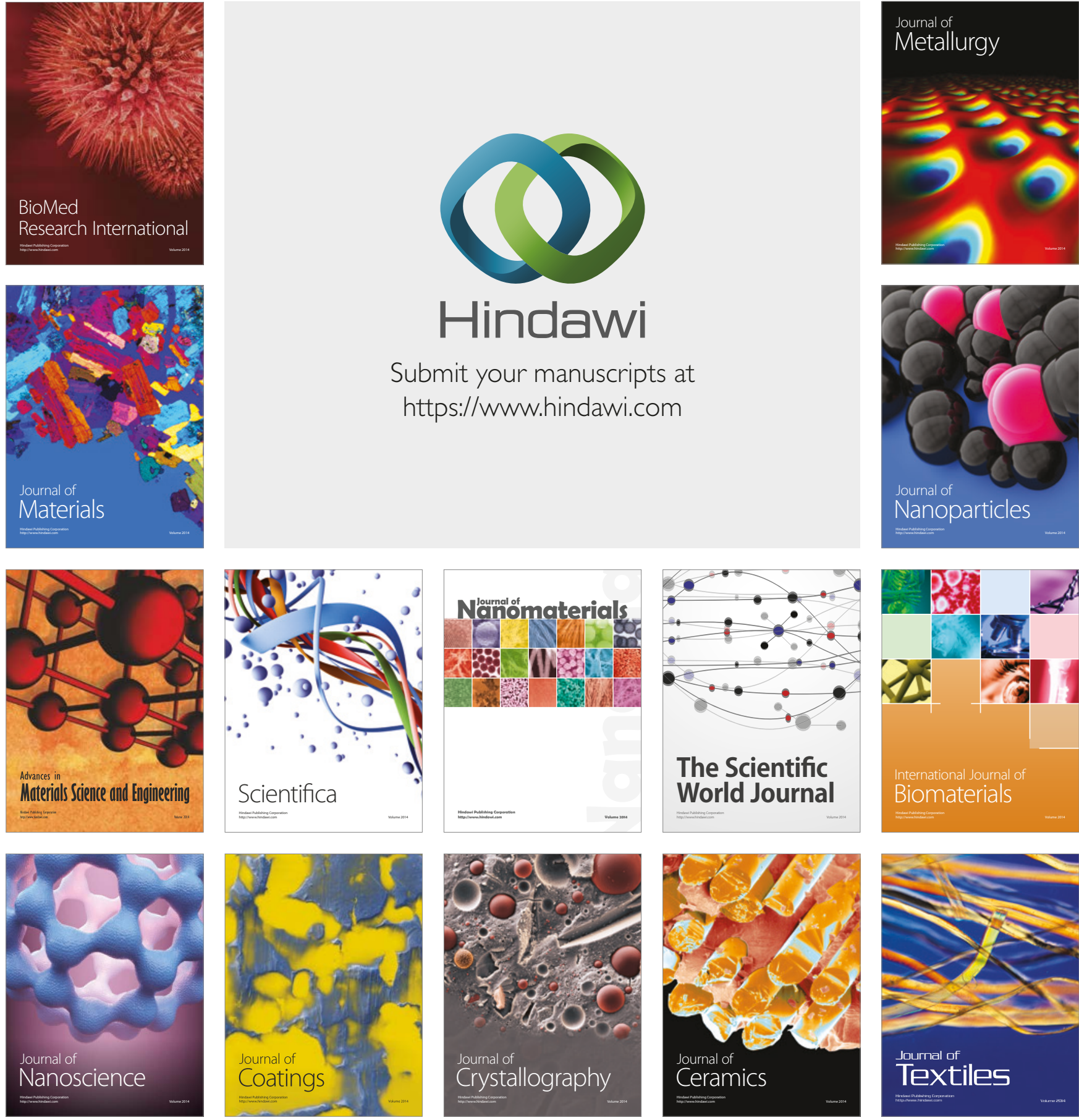

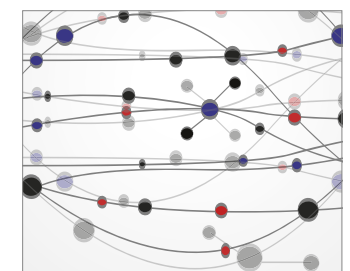

The Scientific World Journal
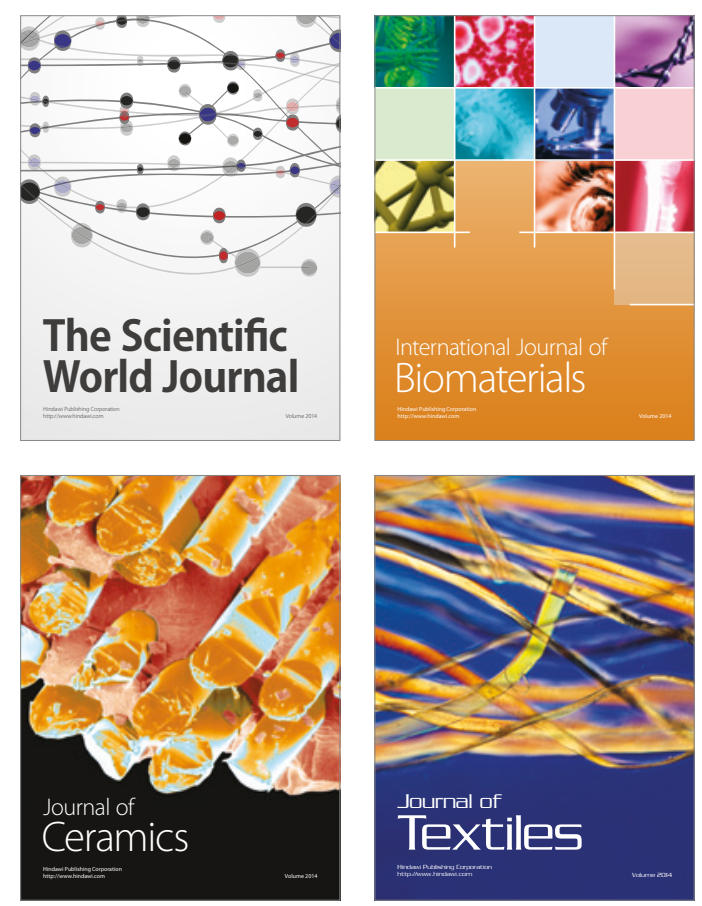